\title{
LincRNA-ROR induces epithelial-to-mesenchymal transition and contributes to breast cancer tumorigenesis and metastasis
}

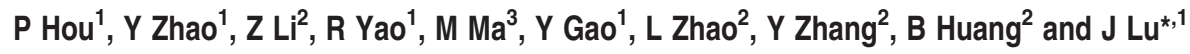

LncRNAs have critical roles in various biological processes ranging from embryonic development to human diseases, including cancer progression, although their detailed mechanistic functions remain illusive. The IncRNA linc-ROR has been shown to contribute to the maintenance of induced pluripotent stem cells and embryonic stem cells. In this study, we discovered that linc-ROR was upregulated in breast tumor samples, and ectopic overexpression of linc-ROR in immortalized human mammary epithelial cells induced an epithelial-to-mesenchymal transition (EMT) program. Moreover, we showed that linc-ROR enhanced breast cancer cell migration and invasion, which was accompanied by generation of stem cell properties. Contrarily, silencing of linc-ROR repressed breast tumor growth and lung metastasis in vivo. Mechanistically, our data revealed that linc-ROR was associated with miRNPs and functioned as a competing endogenous RNA to mi-205. Specifically, linc-ROR prevented the degradation of mir-205 target genes, including the EMT inducer ZEB2. Thus our results indicate that linc-ROR functions as an important regulator of EMT and can promote breast cancer progression and metastasis through regulation of miRNAs. Potentially, the findings of this study implicate the relevance of linc-ROR as a possible therapeutic target for aggressive and metastatic breast cancers.

Cell Death and Disease (2014) 5, e1287; doi:10.1038/cddis.2014.249; published online 12 June 2014

Breast cancer is one of the most common cancers worldwide in women; and distant metastasis is a major cause of poor survival. ${ }^{1}$ In China, the incidence of breast cancer has soared rapidly in recent years. ${ }^{2}$ The epithelial-to-mesenchymal transition (EMT) has been recognized as an important process in normal organ development, as well as in human pathology, including cancer progression. ${ }^{3}$ Increasing evidence implicates that EMT is a crucial step for tumor infiltration and metastasis, and it has been generally accepted that EMT is a key mechanism underlying metastasis in many types of cancer. ${ }^{4,5}$

The long non-coding RNAs (IncRNAs) are RNA molecules over 200 nt in length with little protein-coding potential. ${ }^{6,7}$ Recent studies have revealed that IncRNAs are involved in regulation of multiple biological processes, including development, differentiation and carcinogenesis. ${ }^{8-10}$ LncRNAs have been reported to regulate gene expression by a variety of mechanisms, ${ }^{7}$ for example, HOTAIR functions in epigenetic control of gene expression through interacting with polycomb repressive complex 2 (PRC2)/lysine-specific demethylase complex. ${ }^{10,11}$ In addition, IncRNA H19 acts as a molecular sponge for the let-7 family miRNAs, which are known to have key roles in diverse biological processes. ${ }^{12}$ Recently, it has been reported that the HMGA2 mRNA can function as a competing endogenous RNA (ceRNA) to affect let-7 activity by altering miRNA targeting. ${ }^{13}$ This finding symbolizes the fact that a protein-coding RNA may also function in a similar way as a non-coding RNA. Importantly, recent studies have shown that IncRNAs are involved in a variety of diseases, including breast cancer. ${ }^{14,15}$ However, the specific species of IncRNAs that participate in breast cancer progression remain unknown.

Linc-ROR was first discovered in induced pluripotent stem cells (iPSCs), where it was regulated by the key pluripotency factors Oct4, Sox2 and Nanog. Linc-ROR had a key role in the maintenance of iPSCs and embryonic stem cells (ESCs) by preventing the activation of cellular stress pathways, including the p53 response. ${ }^{16}$ Subsequent studies unveiled that lincROR could act as a p53 repressor in response to DNA damage. ${ }^{17}$ Moreover, recent studies have demonstrated that linc-ROR may function as a molecular sponge for mir-145 to upregulate the mir-145 targets, such as OCT4, SOX2 and Nanog expression. ${ }^{18}$ The fact that many ESCs inducers or repressors often implement a similar role in cancer stem cells (CSCs), for example, mir-205, let-7, SOX2 and OCT4, ${ }^{19-22}$ intrigued us to speculate that linc-ROR may also have a role in cancer progression. Most recently, linc-ROR has been reported to be involved in modulation of hypoxia signaling pathways in hepatocellular carcinoma (HCC) cells. $^{23}$ Despite the implications of linc-ROR in the regulation of a variety of transcription factors and physiological processes,

\footnotetext{
${ }^{1}$ The Institute of Genetics and Cytology, Northeast Normal University, Changchun, China; ${ }^{2}$ The Key Laboratory of Molecular Epigenetics of Ministry of Education (MOE), Changchun, China and ${ }^{3}$ The Breast Surgery, The Tumor Hospital of Jilin Province, Changchun, China

*Corresponding author: J Lu, The Institute of Genetics and Cytology, Northeast Normal University, 5268 Renmin Street, Changchun 130024, China. Tel: +86 431 85098729; Fax: +86 431 85099768; E-mail: luj809@nenu.edu.cn

Abbreviations: EMT, epithelial-to-mesenchymal transition; iPSC, induced pluripotent stem cell; ESC, embryonic stem cell; IncRNA, Iong non-coding RNA; ceRNA, competing endogenous RNA; CSC, cancer stem cell; HCC, hepatocellular carcinoma; FACS, fluorescence activated cell sorter; Rluc, Renilla luciferase; UTR, untranslated region; PTBP1, polypyrimidine tract-binding protein 1; RIP, RNA immunoprecipitation; PRC2, polycomb repressive complex 2; RISC, RNA-induced silencing complex

Received 26.2.14; revised 05.5.14; accepted 07.5.14; Edited by E Candi
} 
the role of linc-ROR in breast cancer metastasis and EMT remains unexplored.

In this study, we identified, for the first time, a role of lincROR in control of EMT and metastasis in breast cancer cells. We showed that linc-ROR overexpression induced EMT in human mammary epithelial cells and resulted in increased CD $44^{\text {high }} / \mathrm{CD} 24^{\text {low }}$ population and potentiated mammosphere-forming ability. In addition, we showed that knockdown of linc-ROR in breast cancer cells repressed tumorigenesis and metastasis in vivo in immunodeficiency mice. Furthermore, we unrivaled a mechanism whereby lincROR regulates EMT by acting as a sponge for mir-205, which is known to have a key role in diverse physiological and pathological processes. We thus identify a novel role and a new regulatory mechanism of linc-ROR in EMT and metastasis in breast cancer.

\section{Results}

Linc-ROR level was positively correlated with human breast cancer. Recent data implicated a link between lincROR and the maintenance and differentiation of ESCs, ${ }^{16,18}$ whereas whether it has a role in cancer progression has not been studied. To evaluate the clinical relevance of linc-ROR in human mammary carcinomas, the linc-ROR expression level in clinical specimens was assessed by quantitative realtime PCR. In a collection of 22 pairs of randomly chosen samples of breast cancer patients, we detected that the lincROR level was significantly higher in cancerous tissues compared with the adjacent non-cancerous tissues (Normal) (Figure 1a), $P=0.0124$. We then measured the expression of linc-ROR in breast cancer cell lines, and we found that lincROR expressed a higher level in breast cancer cell lines (MCF7, BT474, MDA-MB-435, MDA-MB-231, BT549 and MDA-MB-436) than that in the breast epithelia cell MCF10A (Figure $1 \mathrm{~b}$ ). These results suggested that increased level of linc-ROR may be a feature involved in breast tumorigenesis and cancer progression.

Ectopic expression of linc-ROR induced an EMT program in MCF10A cells. To further investigate the function of linc-ROR in breast carcinoma, we established a stable linc-ROR expression MCF10A cell line by using retrovirus infection, and the ectopic overexpression of lincROR was confirmed by RT-PCR (Figure 2a). Compared with the vector-infected cells, linc-ROR-expressing cells became apparently spindle-shaped and began to separate from one another, whereas the vector-infected cells retained their morphology and cell-cell contact (Figure 2b). We then examined the epithelial and mesenchymal markers by using western blotting and immunofluorescence. As can be seen, the epithelial markers E-cadherin and occludin were almost completely lost in linc-ROR-expressing MCF10A cells, whereas the mesenchymal markers vimentin, fibronectin, $\mathrm{N}$-cadherin and $\alpha$-SMA were significantly upregulated in lincROR-expressing MCF10A cells (Figures $2 c$ and d). The realtime PCR assessment of mRNA levels was consistent with that of protein levels (Figure $2 e$ ). To investigate the possible relevance between linc-ROR and other EMT transcription factors and inducers, we assessed the expression of several
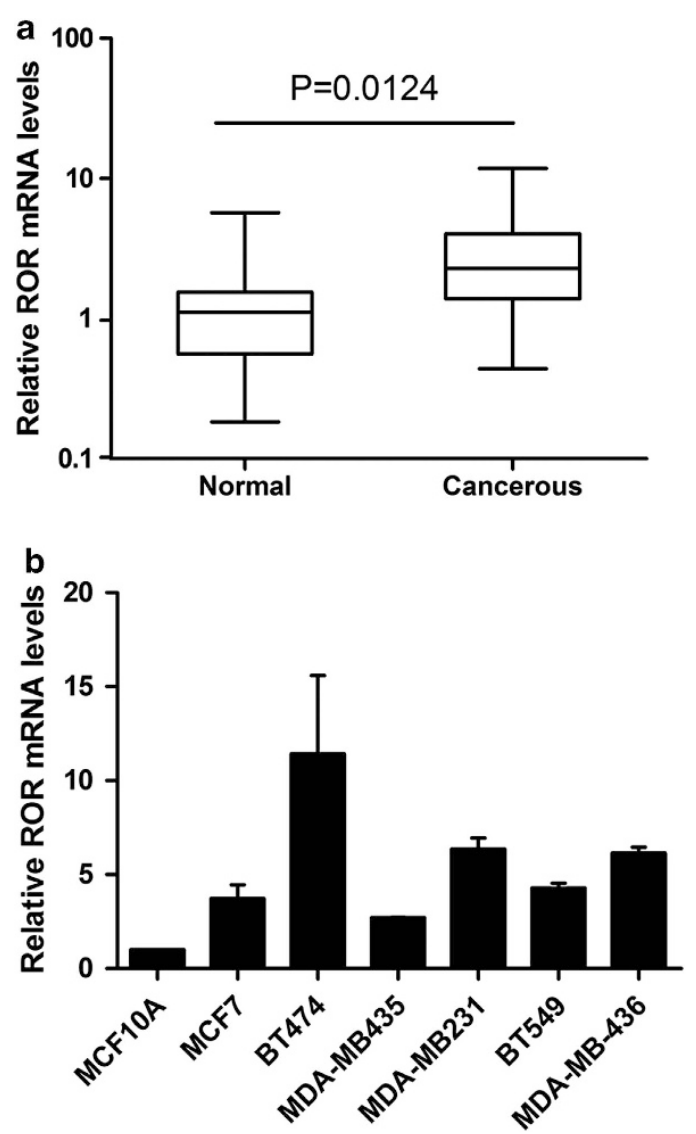

Figure 1 Linc-ROR expression in clinical breast cancer specimens and cancer cell lines. (a) The linc-ROR was significantly upregulated in 22 human breast cancerous tissues compared with the corresponding non-cancerous tissues. The relative linc-ROR mRNA level was normalized to $\beta$-actin. The statistical differences were analyzed using the Paired $t$-test. (b) The linc-ROR expression was upregulated in breast cancer cell lines compared with the immortalized human mammary epithelial cell (MCF10A). The linc-ROR mRNA level was normalized to $\beta$-actin. Error bars represent the mean \pm S.D. of triplicate experiments

transcription factors at both mRNA and protein levels, and we found that the both mRNA and protein expression of ZEB1 and ZEB2 were apparently increased, while the levels of Snail, Slug and Twist were not significantly changed (Figures $2 \mathrm{f}-\mathrm{g}$ ). Thus, both morphological and molecular parameters suggested that linc-ROR may be able to drive the MCF10A cells from an epithelial to mesenchymal status. Next, we ectopically overexpressed linc-ROR in breast cancer cell line MCF7, and similar results were obtained (Supplementary Figures S1a-c). Meanwhile, loss-of-function experiment with linc-ROR shRNAs was performed, and the silencing efficiency and specificity of each shRNAs in knockdown of linc-ROR mRNA level were confirmed in MDA-MB-231 cells (Supplementary Figure S1d). The results showed that partial loss of endogenous linc-ROR expression in MDA-MB-231 cells prominently increased the protein expression of E-cadherin and partly decreased the vimentin level (Supplementary Figure S1e). Further, a BrdU incorporation staining assay was carried out to evaluate the effect of lincROR on cell proliferation, and the results showed that neither the overexpression nor the silencing of linc-ROR had significant effects on cell proliferation (Supplementary 
a

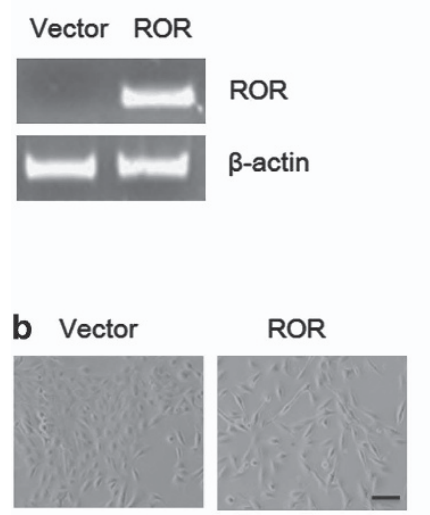

d
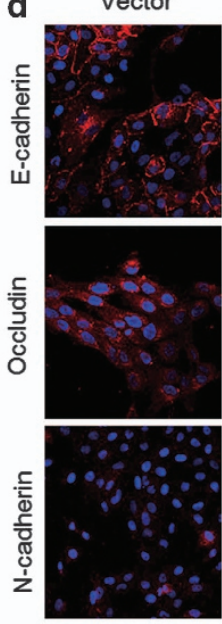

ROR
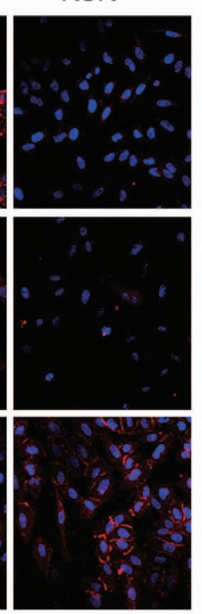
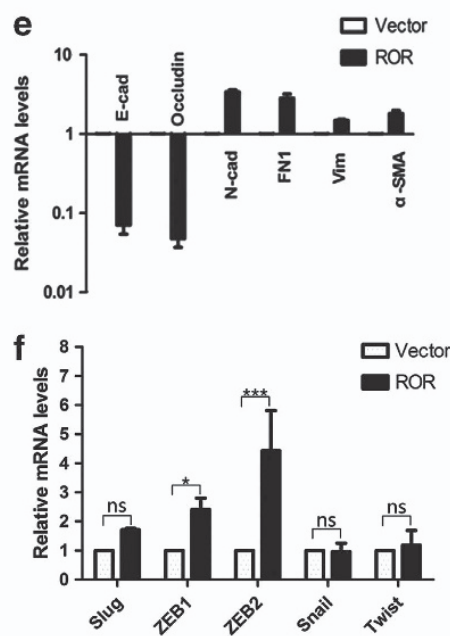

Fibronectin

$\mathrm{N}$-cadherin

Occludin

E-cadherin

$\beta$-actin

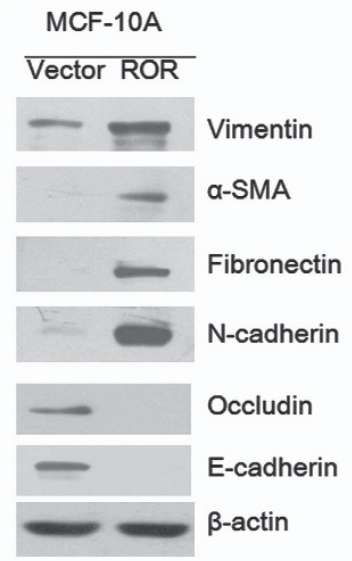

Vector
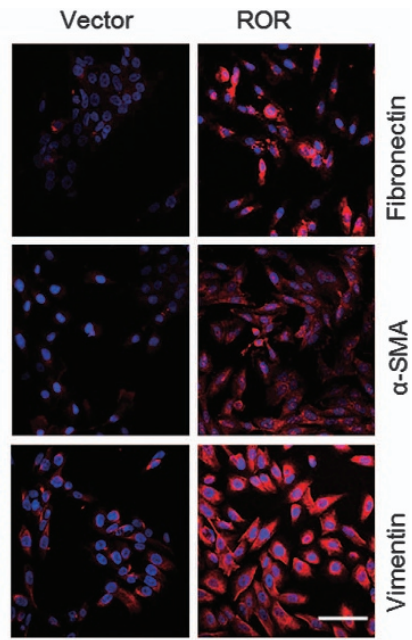

g

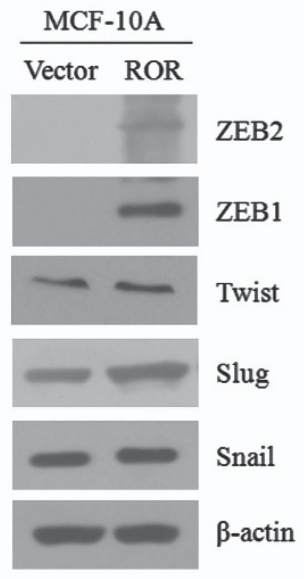

Figure 2 Ectopic expression of linc-ROR in MCF10A cells induced an EMT program. (a) Ectopic expression of linc-ROR mRNA expression was confirmed by RT-PCR after retrovirus infection in MCF10A cells. (b) The morphology of MCF10A cells expressing the control vector or linc-ROR was examined by phase-contrast microscopy. Scale bar, $100 \mu \mathrm{m}$. (c) Immunoblotting analysis of the epithelial (E-cadherin and Occludin) and mesenchymal markers (Fibronectin, N-cadherin, Vimentin and $\alpha$-SMA) in MCF10A cells expressing linc-ROR or the control vector. (d) Immunofluorescence staining for the EMT makers. Scale bar, $100 \mu \mathrm{m}$. (e) Expression of EMT marker mRNAs were assessed by real-time PCR. (f) The mRNA expression levels of EMT inducers (Snail, Slug, Twist, ZEB1 and ZEB2) were assessed by real-time PCR in linc-ROR or the control vector expressing MCF10A cells. (g) Immunoblotting analysis of EMT inducers (Snail, Slug, Twist, ZEB1 and ZEB2) in MCF10A cells expressing linc-ROR. The mRNA levels were normalized to $\beta$-actin. Error bars represent the mean \pm SD of triplicate experiments. Statistical differences were analyzed using the Paired $t$-test $\left({ }^{* \star *} P<0.001,{ }^{*} P<0.05\right.$, non-significant (ns) $P>0.05)$

Figure S1f). Together, these results suggest that linc-ROR may be a novel EMT inducer, and it has a role in breast cancer progression.

\section{Linc-ROR promoted migration and invasion in breast} cancer cells. We next investigated the biological effects of linc-ROR in breast cancer cell migration and invasion. The wound-healing assay showed that overexpression of lincROR increased MCF10A cell mobility (Figure 3a, left), whereas reduction of linc-ROR inhibited MDA-MB-231 mobility (Figure $3 \mathrm{a}$, right). In addition, transwell assay revealed that linc-ROR overexpression remarkably increased the migration ability (Figure $3 \mathrm{~b}$, left, $P<0.01$ ), whereas knockdown of linc-ROR decreased the migration (Figure $3 \mathrm{~b}$, right, $P<0.01$ ) in MDA-MB-231 cells. Similarly, invasion assay demonstrated that linc-ROR promoted the invasion ability (Figure 3c, left, $P<0.01$ ) and loss of ROR repressed the invasion ability (Figure $3 \mathrm{c}$, right, $P<0.01$ ) in MDA-MB231 cells. These results indicated that linc-ROR was able to significantly promote the invasion and migration of breast cancer cells.

The linc-ROR-induced EMT program generated stem-like cells. Previous evidence points to a link between EMT and CSC phenotype, and EMT program has been shown to be frequently accompanied by the acquisition of increased CD44 $4^{\text {high }} / \mathrm{CD} 24^{\text {low }}$ population and mammosphere-formation ability. ${ }^{24}$ We thus intended to determine whether linc-ROR can affect the CSC population and the self-renewal capacity. Our fluorescence activated cell sorter (FACS) analysis demonstrated that the stem cell phenotype CD44 ${ }^{\text {high/ }}$ CD24 ${ }^{\text {low }}$ subpopulation was elevated in MCF10A-ROR cells (Figure 4a). Meanwhile, as shown in Figures $4 \mathrm{~b}$ and $\mathrm{c}$, the MCF10A-ROR cells formed more and bigger 
a

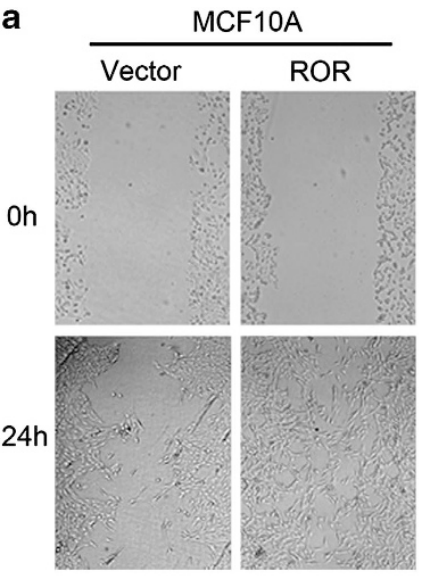

b

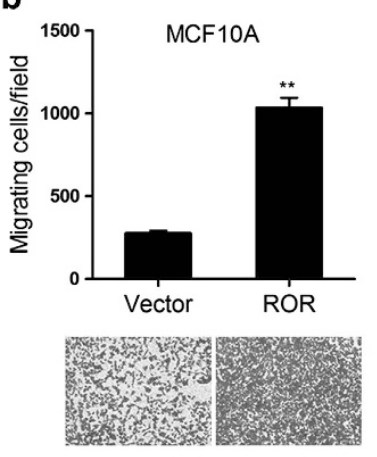

C

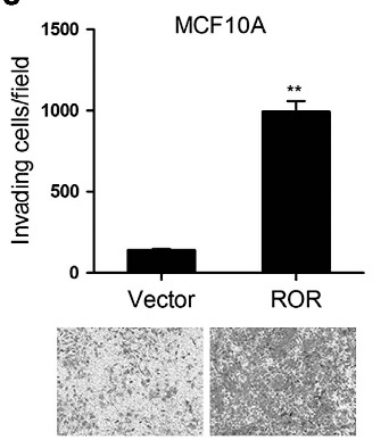

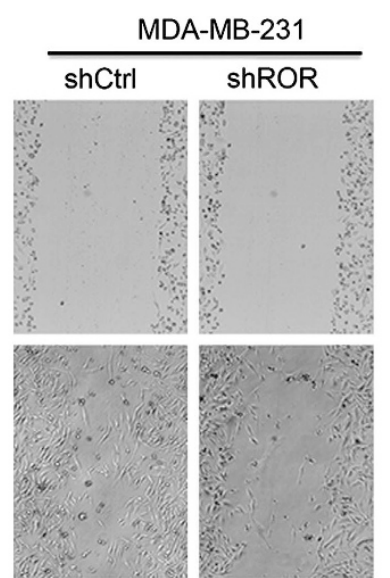
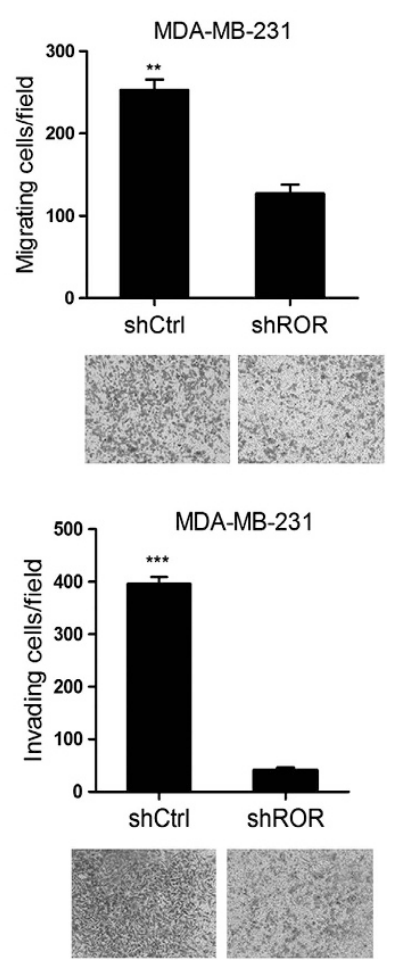

Figure 3 Effects of linc-ROR on invasion and migration in breast cancer cells. (a) Wound-healing assay to assess the effect of linc-ROR on cell mobility in MCF10A and MDA-MB-231 cells. (b) The effect of linc-ROR on migration ability was measured using transwell assays in MCF10A and MDA-MB-231 cells. (c) The effect of linc-ROR on invasion ability. The data represent the mean number of cells per field and are presented as the means \pm S.D. $\left({ }^{* *} P<0.01,{ }^{* \star *} P<0.001\right)$. All the experiments were repeated three times

mammospheres compared with the vector-infected MCF10A cells. We thus concluded that the linc-ROR-induced EMT process was accompanied by the generation of stem-like cells and an elevated self-renewal capacity in breast CSCs.

Linc-ROR promoted breast cancer tumorigenesis and metastasis in vivo. EMT process has often been implicated in cancer cell dissemination and tumor metastasis. ${ }^{3}$ To further evaluate the physiological and pathological relevance of linc-ROR upregulation in breast cancer, we investigated the functional role of linc-ROR in tumorigenesis

and metastasis in vivo. First, we subcutaneously injected one million breast epithelial MCF10A cells that exogenously express linc-ROR into nude mice; however, no tumors were formed in either MCF10A-vector or MCF10A-ROR cells after 10 weeks (data not shown). Then, to determine the function of endogenous linc-ROR in tumor growth, nude mice were subcutaneously transplanted with either MDA-MB-231-shCtrl or MDA-MB-231-shROR cells, and we found that the control MDA-MB-231-shCtrl cells formed apparently larger tumors than the mice injected with MDA-MB-231-shROR cells (Figure 5a), indicating that the endogenous linc-ROR affected tumor growth. Furthermore, we examined the effects of linc-ROR on lung metastasis using the linc-ROR knockdown cell lines. Cells were injected via the lateral tail veins of 4-5-week-old nude mice. After 9 weeks, the mice injected with MDA-MB-231-shCtrl cells metastasized more efficiently than that injected with linc-ROR knockdown MDAMB-231-shROR cells, as judged by the percentage of mice that developed lung nodules and the number of lung nodules per mouse (Figures $5 b$ and c). Subsequently, the lung tissue sections were prepared and stained with hematoxylin and eosin before examination under a microscope, and we detected smaller and fewer metastatic foci in the samples from mice injected with MDA-MB-231-shROR cells than that with control cells (Figure $5 \mathrm{~d}$ ). These data further indicate that linc-ROR may have a pivotal role in breast cancer tumorigenesis and metastasis.

Linc-ROR was associated with miRNPs and acted as a molecular sponge for mir-205. There have been indications that LncRNAs can act as molecular sponges to bind specific miRNAs to interfere in their function. ${ }^{12}$ Also, recent studies have revealed that linc-ROR is enriched in the cytoplasm and functions as a sponge for mir-145. ${ }^{18}$ Our bioinformatic analysis revealed the putative complementary sequences for the seed region of mir-205 and for mir-34a and let-7 in linc-ROR (Figure 6a). Mir-205 has been implicated to function as a tumor repressor targeting the ZEB1, ZEB2, ErbB3 and VEGF-A and can negatively regulate the stem cell ability. $^{19,25}$ Also, mir-34a and let-7 have been shown to suppress the breast CSC properties. ${ }^{20,26,27}$ These data point to the possible association between linc-ROR and miRNAs for its function.

To validate this speculation, we used a psiCHECK2 reporter plasmid to test the relationship between linc-ROR and miRNAs. We cloned the linc-ROR full-length transcript or ZEB2 $3^{\prime}$-UTR (untranslated region) into the $3^{\prime}$-UTR of Renilla luciferase (Rluc) gene of psiCHECK2. The psiCHECK2 plasmid also contains a downstream constitutively expressed firefly luciferase gene as an internal control for normalization. We then showed that transient co-transfection of mir-205-5p mimics into HEK293T cells inhibited the Rluc activity in a dose-dependent manner (Figure $6 b$ ), while the mir-34a-5p and let-7a-5p mimics had little influence on Rluc activity of psiCHECK2-ROR (Figure 6c). To determine whether linc-ROR acts as a sponge to mir-205, we transfected psiCHECK2-ZEB2 into HEK293T cells together with the mir-205-5p mimics and increasing amounts of linc-ROR, and we detected that the Rluc activity increased in response to linc-ROR in a dose-dependent manner (Figure 6d). Together, 
a

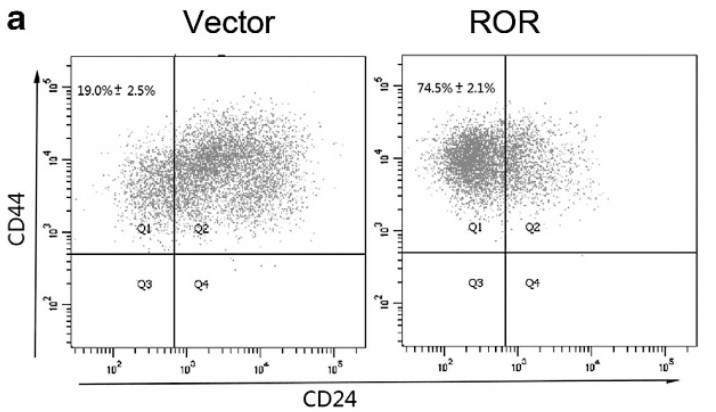

b
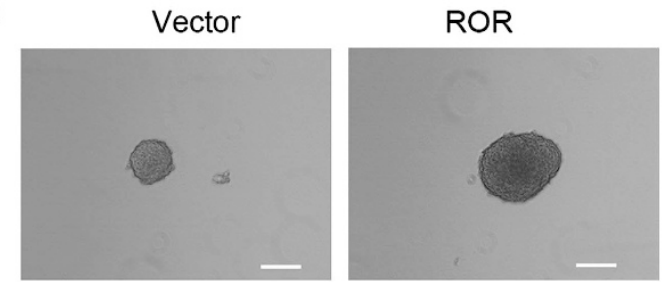

C

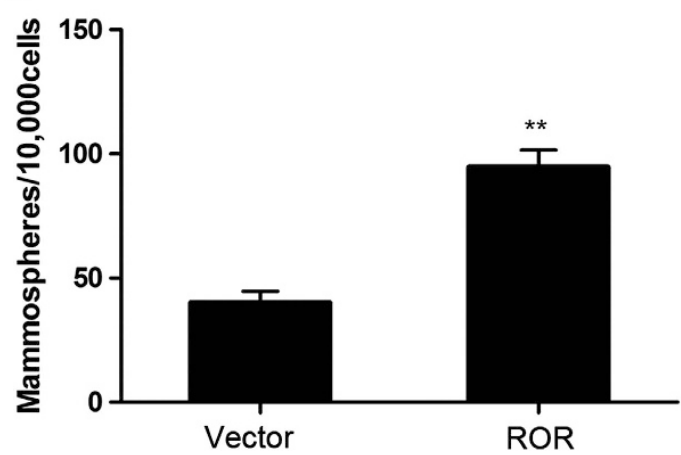

Figure 4 The linc-ROR-induced EMT generated stem cell-like phenotype in mammary epithelial cells. (a) Flow cytometric evaluation of $\mathrm{CD} 44^{\text {high }} / \mathrm{CD} 24^{\text {low }}$ subpopulation in MCF-10A cells expressing vector or linc-ROR. The percentages of mean $\mathrm{CD} 44^{\text {high }} / \mathrm{CD} 24^{\text {low }}$ population \pm S.D. of triplicate experiments are indicated. (b) Reprehensive images of mammospheres formed from MCF10A cells. Scale bar, $100 \mu \mathrm{m}$. (c) The quantification of mammosphere numbers formed from MCF10A cells in independent experiments (error bar, means \pm S.D., ${ }^{* \star} P<0.01$ )

these results strongly point to a role of linc-ROR as a molecular sponge for mir-205, and presumably linc-ROR may regulate EMT program through preventing degradation of mir-205 targets (ZEB1 and ZEB2). This was in accordance with the ZEB1 and ZEB2 expression levels after linc-ROR/mir205 overexpression or silencing (Figure 1f, Supplementary Figures S3a and b). Our data also showed that several ZEB1/ 2-related miRNAs such as mir-205 and mir-200 family members were downregulated in linc-ROR-overexpressing cells (Supplementary Figure S3c). Thus, we conclude that linROR functions as a molecular sponge for mir-205 to affect mir-205 targets in the process of EMT.

The cytoplasmic miRNAs are usually assembled into ribonucleoprotein complexes termed miRNPs, which have important roles in target $m R N A$ recognition and in translational repression. ${ }^{28}$ Ago2, a core component of the RNA-induced silencing complex (RISC), is also a component of miRNPs. ${ }^{29}$ Recent studies have shown that IncRNAs can act as a molecular sponge to regulate the miRNA activity by associating with miRNPs. ${ }^{12,30}$ To further verify the role of linc-ROR as a molecular sponge, we next carried out the RNA immunoprecipitation (RIP) analysis with MS2-binding protein (MS2bp), which specifically binds RNAs that contain MS2binding sequences (MS2bs) when they are co-expressed. We constructed vectors expressing linc-ROR combined with MS2bs elements (ROR-MS2bs), and we co-transfected ROR-MS2bs, Flag-MS2bp, mir-205 and mir-200a (a negative control) into HEK293T cells. Subsequently, we performed RIP assay using the ANTI-FLAG M2 Affinity Gel. Real-time PCR assays showed that the mir-205 was enriched in ROR$12^{*}$ MS2bs RNA in contrast to the negative control MS2bs RNA (Figure 6e). We then confirmed that linc-ROR was associated with AGO2 (Figure 6f), a result consistent with a recent study. ${ }^{18}$ Additionally, our RIP assays revealed that lincROR was specifically recruited to AGO2 (Figure 6g). Furthermore, we also estimated the role of PTBP1 (polypyrimidine tract binding protein 1; a linc-ROR binding protein) ${ }^{17}$ in lincROR-induced EMT, and we found that linc-ROR did not regulate gene expression through PTBP1 (Supplementary Figure S2) in our experiment model. These data indicate that linc-ROR interacts with miRNPs and functions as a ceRNA to regulate the miRNA activity in breast cancer progression.

\section{Discussion}

Linc-ROR is a large intergenic non-coding RNA about $2600 \mathrm{nt}$ in length and has been first described in iPSCs and shown to have a role in ESC generation. ${ }^{16,18}$ Although linc-ROR had multiple functions, its role in breast cancer remains unclear. In this study, we provide evidence that lin-ROR is a critical positive regulator of EMT; it potentiates breast cancer cell migration and invasion in vitro and promotes tumor metastasis in immunodeficient mice in vivo. We also discover that linc-ROR promotes the generation of stem-like cells. Mechanistically, we demonstrate that linc-ROR functions as a ceRNA to regulate mir-205 activity to prevent the mir-205 target genes, such as ZEB1 and ZEB2, from degradation (Figure 6d).

Significantly, this study identifies the IncRNA linc-ROR as an inducer of EMT in breast cancer cells. EMT has a central role in embryogenesis ${ }^{31,32}$ and is also involved in acquisition of malignant traits in carcinoma cells. ${ }^{3}$ In recent years, it has been found that the embryonic transcription factors confer many malignant traits on the carcinoma cells, including the motility, invasiveness and resistance to apoptosis. ${ }^{33-35}$ It has become increasingly apparent that IncRNAs are important regulators of a wide range of biological processes, including embryogenesis and cancer procession. ${ }^{8,9}$ Up to recent years, about 1000 IncRNAs have been identified in embryogenesis and in ESCs, ${ }^{36,37}$ while just a few of them have been studied in cancers. Among these few examples, for instance, H19 IncRNA was originally shown to have important roles in embryonic development and in growth control and is highly expressed during embryogenesis but significantly downregulated after birth. ${ }^{38,39}$ Recent studies have implicated that $\mathrm{H} 19$ can also function as an oncogene. ${ }^{40-42}$ These data suggest the similar roles of IncRNAs in both ESCs and in cancer progress. In this study, we identify that linc-ROR is correlated with breast cancer tumorigenesis and metastasis. 
a

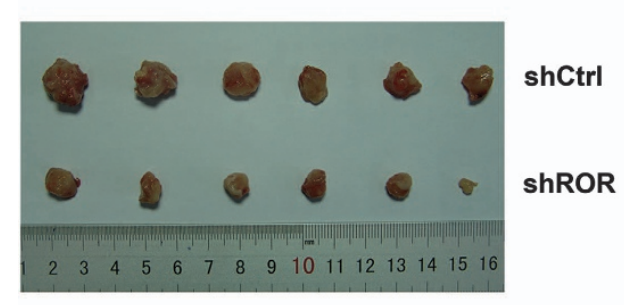

b

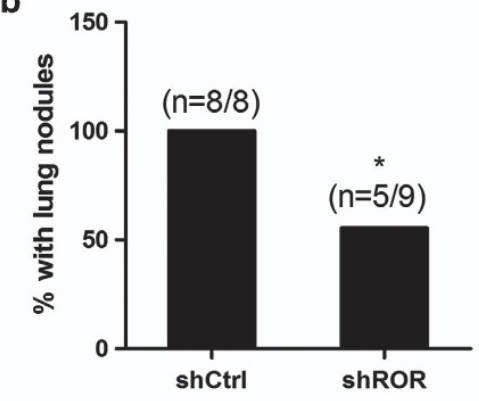

d

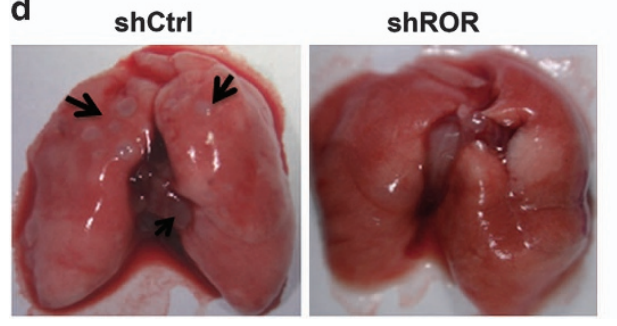

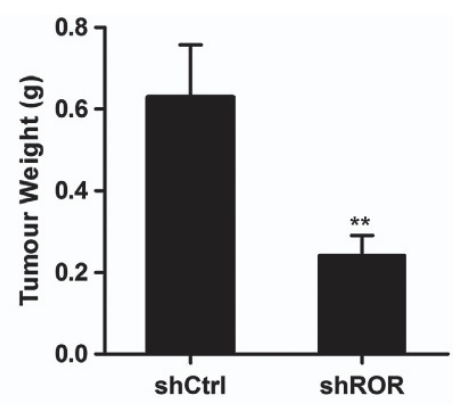

c
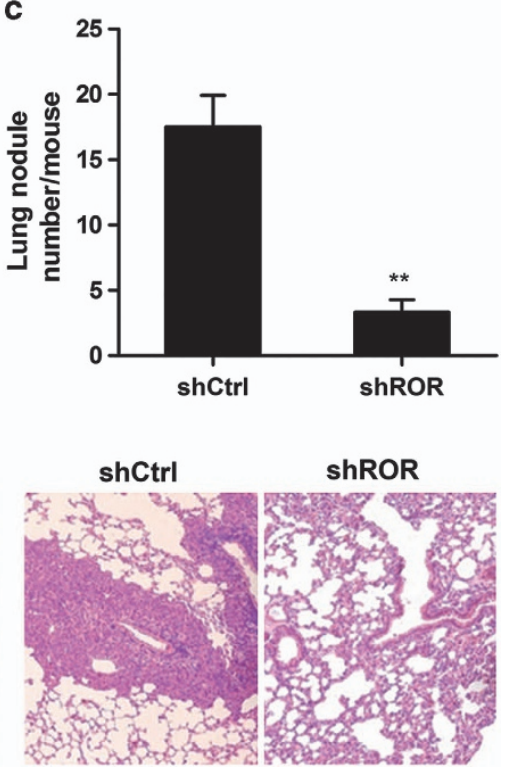

Figure 5 Knockdown of linc-ROR inhibited tumorigenesis and metastasis in nude mice. (a) Stable shCtrl or shROR-infected MDA-MD231 cells were subcutaneously injected into BALB/c female nude mice ( $n=6$, for each experimental group), and 8 weeks later, the xenograft tumors were peeled off and weighted. The tumor weights are presented as the means \pm S.D., ${ }^{* \star} P<0.01$. (b) Linc-ROR knockdown MDA-MB-231 cells were injected via the lateral tail veins. Lung nodules were analyzed as percentage of mice that developed lung metastases after 9 weeks. Numbers over the bars indicate metastasis incidence. ${ }^{*} P<0.05, \chi^{2}$ test. (c) Lung nodules at week 9 were analyzed as the numbers of nodules per mouse. ${ }^{\star \star} P<0.01$, Student's $t$-test. (d) Representative lung images at week 9 , corresponding hematoxylin-eosin-stained lung sections are shown

Our study, as well as others, strongly points to a close link between the roles of IncRNAs in embryogenesis and in cancer progression.

It has been established that the function of IncRNAs often depends on the proteins they conjoined, for example, Xist, Air and HOTAIR have been found to interact with chromatin remodeling proteins PRC2 complex and G9a to mediate gene regulation. ${ }^{10,43,44}$ Under the DNA damage conditions, lincROR functions as a negative regulator of p53 through interaction with heterogeneous nuclear ribonucleoprotein I (hnRNP I, also named PTBP1) in MCF7 cells. ${ }^{17}$ PTBP1 was previous reported to be involved in multiple biological processes such as cell cycle, cell proliferation and tumorigenesis. ${ }^{45-47}$ In this study, we unraveled that silencing of the PTBP1 expression with shRNA in linc-ROR-overexpressing MCF10A cells resulted in partial reversion of the EMT phenotypes, including the increased E-cadherin, and decreased N-cadherin and vimentin (Supplementary Figures $\mathrm{S} 2 \mathrm{a}$ and b). Furthermore, we examined the subcellular localization of PTBP1 by immunofluorescence, and detected the enrichment of PTBP1 in nuclei (Supplementary Figure $\mathrm{S} 2 \mathrm{c})$. As linc-ROR was reported to be mainly located in cytoplasm, ${ }^{17,18}$ it would be likely that linc-ROR does not regulate gene expression through PTBP1, although PTBP1 may function in EMT process as shown above in our study. Although PTBP1 would shuttle between the nucleus and cytoplasm under stress conditions, such as hypoxia, ${ }^{48}$ whether PTBP1 was involved in linc-ROR-induced EMT in other conditions needs to be further investigated.

CSCs are characterized by a high CD $44^{\text {high }} / \mathrm{CD} 24^{\text {low }}$ antigenic phenotype, as discovered, for example in breast cancers. ${ }^{49}$ Recent evidence indicates that an EMT program may generate cells with stem cell properties. ${ }^{24}$ Our data from this study showed that ectopic expression of linc-ROR in MCF-10A cells predominantly elevated the percentage of CD $44^{\text {high }} /$ CD2 $4^{\text {low }}$ subpopulation and increased the efficiency of mammosphere-formation ability (Figure 3).

It has been reported that ZEB1 and ZEB2 are the key regulators for maintaining the stem cell properties. The transcription factors ZEB1 and ZEB2 are able to initiate an EMT program through downregulation of E-cadherin transcription by repressing promoter activity. ${ }^{50}$ Moreover, ZEB1 and ZEB2 are regulated by mir-200 family and mir205, and the stem cell-like properties are strongly associated with the 
a

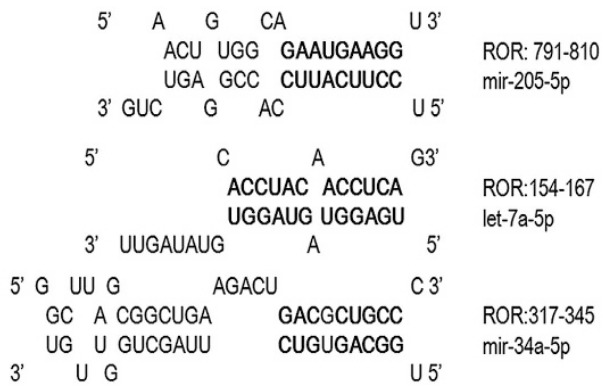

b
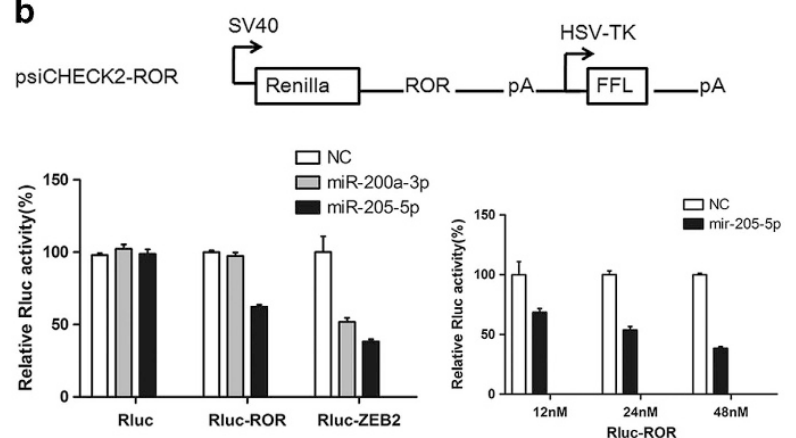

e
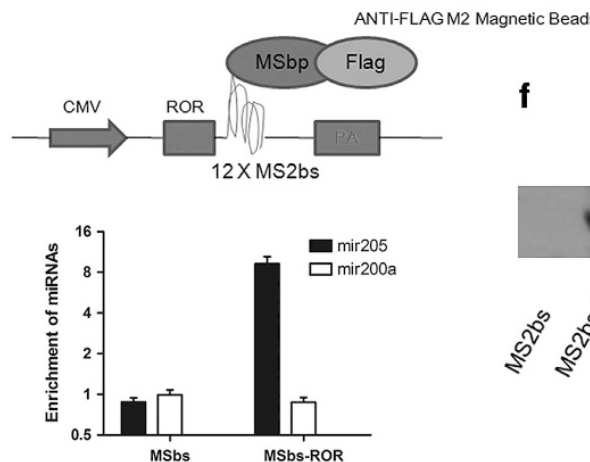

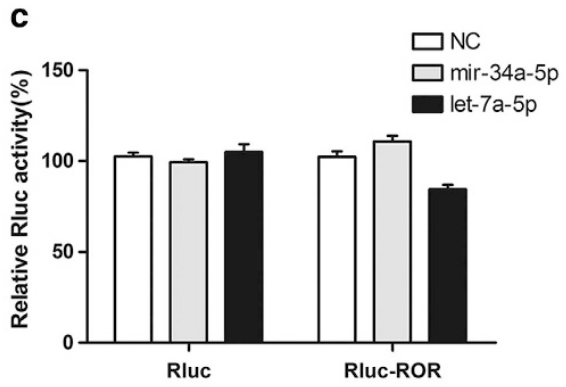

d

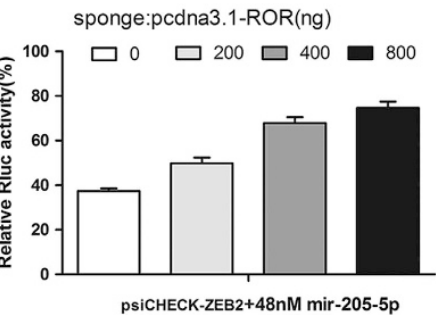

g

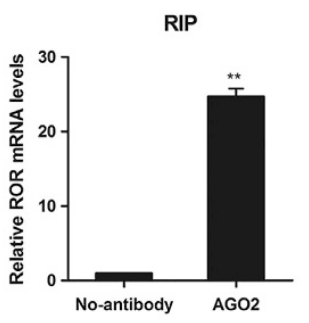

Figure 6 Linc-ROR was associated with miRNPs and acted as a molecular sponge for mir-205. (a) Prediction for miRNA-binding elements on linc-ROR by Miranda. (b) The indicated reporter constructs were transfected into $293 \mathrm{~T}$ cells, together with control miRNA (NC), mir-205-5p or mir-200a-5p, at a final concentration of $48 \mathrm{nM}$, and additional detection of the indicated Rluc activity at a final concentration of 12,24 and $48 \mathrm{nM}$. Numbers are mean \pm S.D. $(n=3)$. (c) Detection of the indicated Rluc activity affected by mir-34a-5p or let-7-5p at a final concentration of $48 \mathrm{nM}$. (d) Co-expression of linc-ROR rescued the relative Rluc activity of reporters containing ZEB2 when co-transfected with mir-205-5p. (e) The binding ability of linc-ROR transcript to mir-205-5p that was precipitated by cDNA combined with MS2-binding sequences (MS2bs) and its binding protein Flag-MS2bp. Measured by real-time PCR. Numbers represent mean \pm S.D. $(n=3)$ (f) Immunoblotting detection of AGO2 after Flag-MS2bp-MS2bs-based pull-down assay. ANTI-FLAG M2 Affinity Gel was used for pull-down assay. (g) RIP assay detection of the linc-ROR-binding efficiency to AGO2 in MCF10A-ROR cells. Error bars represent the mean \pm S.D. of triplicate experiments, ${ }^{\star *} P<0.01$

expression of miR-205 and miR-200 families. ${ }^{19,51,52}$ In relevance to these data, we showed that linc-ROR acted as a molecular sponge for mir-205 to protect the mir-205 targets from degradation (Figure 6d). Additionally, we found that the expression levels of mir-205 members were decreased upon linc-ROR overexpression in MCF10A cells (Supplementary Figure S3c), suggesting that linc-ROR may also decrease mir205 expression through the formation of an RISC complex. Apart from the mir-205 members, apparent reduction of the levels of mir-200 family members in linc-ROR-overexpressing MCF10A cells was also detected (Supplementary Figure S3c), probably as a consequence of the upregulation of ZEB1 and ZEB2, because no binding between mir-200 family members and linc-ROR was found, and the mir-200 family members have little effect on the Rluc activity of psiCHECK2ROR (data not shown). Most recently, studies have shown that linc-ROR modulates hypoxia signaling pathways through regulating the activity of mir-145 in HCC cells; ${ }^{23}$ our data has also shown that mir-145 expression level was decreased in linc-ROR induced EMT process (Supplementary Figure S3c), which suggested linc-ROR may function through regulating multiple miRNAs. Conclusively, our data support a model in which linc-ROR regulates the EMT process of breast cancer cells mainly via sponge mir-205, although other possible mechanisms cannot be ruled out. This model provides some new insights into regulatory machineries in EMT program, especially in association with the tumorigenesis and cancer progression. 
In this study, we investigated the role of linc-ROR in EMT process in breast cancer. We showed that expression of linc-ROR was higher in breast cancer tissues than that in adjacent normal breast tissues, and higher linc-ROR expression was linked with metastasis in breast cancer (Figure 1a, Supplementary Figure S4a). In addition, linc-ROR may have close relationship with the EMT-related factors (E-cadherin, ZEB2, mir-205, mir-145), as we detected lower expressions of E-cadherin, mir-205 and mir-145 in breast cancer tissues and higher ZEB2 expression in breast cancer tissues (Supplementary Figures S4b-e). These data implicate that linc-ROR may act as a prognostic factor in breast cancer.

To summarize, this study identifies the IncRNA linc-ROR as a novel potential oncogene in breast cancer through induction of an EMT program. Our observations that silencing of lincROR in aggressive breast cancer cells dramatically blocked tumor growth and metastasis in vivo raise the possibility for the development of linc-ROR as a potent target for new therapies for the highly aggressive and malignant breast cancers in clinical practice.

\begin{abstract}
Materials and Methods
Specimens and cell lines. Breast tissue specimens were obtained from the Tumor Hospital of Jilin Province, China. Samples were frozen in liquid nitrogen immediately after surgical removal. All human tissues were collected using the protocols approved by the Ethics Committee of the Jilin Tumor Hospital. All the cell lines were obtained from the American Type Culture Collection (ATCC, Manassas, VA, USA). MCF10A cells were cultured in DMEM/F12 (Sigma, St. Louis, MO, USA) supplemented with $5 \%$ horse serum (Gibco, Grand Island, NY, USA), $20 \mathrm{ng} /$ $\mathrm{ml}$ EGF (R\&D), $0.5 \mathrm{mg} / \mathrm{ml}$ hydrocortisone (Sigma), $100 \mathrm{ng} / \mathrm{ml}$ cholera toxin (Sigma), $10 \mathrm{mg} / \mathrm{ml}$ insulin (Gibco) and pen/strep. MDA-MB-231 cells were cultured in Leibovitz's L-15 medium with $10 \% \mathrm{FBS}$ at $37^{\circ} \mathrm{C}$ without $\mathrm{CO}_{2}$. BT549, BT474, MDA-MB-436 and MDA-MB-435 cells were cultured in RPMI-1640 (Sigma) medium supplemented with 10\% FBS (ExCell Bio, Shanghai, China). HEK293T cells were cultured in DMEM (Sigma) supplemented with 10\% FBS.
\end{abstract}

Plasmids and virus infection. The retrovirus expression plasmid pBabepuro-ROR was a gift from Dr. Sabine Loewer (Children's Hospital Boston, Boston, MA, USA). The pcDNA3-Flag-MS2bp and pcDNA3-12xMS2bs plasmids were provided by Dr. Xiaofei Zheng (Academy of Military Medical Sciences, China). The cDNA of linc-ROR was inserted into the ECOR1 site of pcDNA3-12 $\times$ MS2bs vector. The human linc-ROR and PTBP1 shRNA were cloned into pEN_hH1c and then transferred into destination vector pDSL_hpUGIP (ATCC) by LR-clonase (Invitrogen, Camarillo, CA, USA). The ROR full length and ZEB2 $3^{\prime}$-UTR fragments were obtained by RT-PCR and were inserted into the luciferase reporter vector psiCHECK2 (Promega, Fitchburg, WI, USA) between Xho1 and Not1 sites. The shRNA and plasmid cloning primer sequences are listed in Supplementary Table S1. Retroviruses were generated by co-transfecting 293T cells with $\mathrm{pBabe-}$ puro-ROR and the packaging plasmids (pUMVC and pVSV-G); the supernatants were collected $48 \mathrm{~h}$ later, filtered through $0.45-\mu \mathrm{m}$ filters (Millipore, Temecula, CA, USA) and concentrated using Amicon Ultra centrifugal filters (Millipore 100KD MWCO). The concentrated virus was used to infect MCF10A and MCF7 cells. Stable cell lines were selected with $2 \mu \mathrm{g} / \mathrm{ml}$ puromycin for about 2 weeks. Lentiviruses were produced by co-transfecting 293T cells with one of the shRNA expression plasmids and packaging plasmids (psPAX2 and pMD2.G), and then lentiviruses were obtained using the same process as for retroviruses described above. Stable linc-ROR or PTBP1 knockdown cells were generated by lentivirus infection.

RNA extraction, RT-PCR and real-time PCR analysis. Total RNA was extracted from breast tissue specimens or cells using the Trizol reagent (Invitrogen) following manufacturer's instructions. The cDNA was generated with random primers (Promega) using the Reverse Transcription System (Promega) or miRNA RT-PCR Kit (Takara, Shiga, Japan). Real-time PCR was carried out on a Roche LightCycler480 using SYBR Green Realtime PCR Master Mix (TOYOBO, Osaka, Japan). PCR reactions were run in triplicate for three independent experiments. U6 snRNA or $\beta$-actin was used as an internal control. The sequences of PCR primers are listed in Supplementary Table S1.

Immunoblotting. Cells were washed twice in cold PBS and lysed in RIPA lysis buffer $(50 \mathrm{mM}$ Tris- $\mathrm{HCl}, \mathrm{pH} 7.4,150 \mathrm{mM} \mathrm{NaCl}, 1 \%$ sodium deoxycholate, $1 \%$ Triton X-100 and $0.1 \%$ SDS) plus protease inhibitor cocktail (Roche, Mannheim, Germany). Protein lysates were subjected to SDS-PAGE, transferred to PVDF membrane (Millipore) and detected with appropriate primary antibodies coupled with HRP-conjugated secondary antibodies by ECL reagent (GE Healthcare, Buckinghamshire, UK). Primary antibodies against the following proteins were used: E-cadherin, N-cadherin, Vimentin, Fibronectin (BD Biosciences, San Jose, CA, USA); Occludin (Invitrogen); $\alpha$-SMA, Twist (Sigma); Snail, Slug (Abcam, Hong Kong, China); $\beta$-actin (Tianjin Sungene Biotech, Tianjin, China); and ZEB1, ZEB2, AGO2, PTBP1 (Santa Cruz, Dallas, TX, USA). Secondary antibodies used were: HRP-goat anti-mouse, HRP-goat anti-rabbit, and HRP-rabbit anti-goat (ZSGB-BIO, Beijing, China).

Immunofluorescence. Cells seeded on glass coverslips in 24-well plates were fixed in $4 \%$ formaldehyde solution and permeabilized with $0.2 \%$ Triton X-100/ PBS. Cells were blocked with $5 \%$ bovine serum albumin for $1 \mathrm{~h}$ and incubated with primary antibody at room temperature for $1 \mathrm{~h}$, followed by incubation with TRITCconjugated secondary antibodies (Invitrogen) for $1 \mathrm{~h}$, and then stained with DAPI. Finally, images were taken under a confocal microscope (OLYMPUS, Tokyo, Japan).

Wound healing, transwell migration and invasion assays. For wound-healing assay, cells were seeded at a density of $1 \times 10^{6}$ cell/well onto sixwell plates. Sixteen hours later, cell layers were wounded using a sterile $10-\mu l$ pipette tip; the suspended cells were washed away with PBS, and then the cells were cultured in medium with $1 \%$ FBS. Gap areas were photographed with a light microscope at different time periods. In vitro cell migration and invasion assays were performed using 24-well inserts transwell chambers $(8.0 \mu \mathrm{m}$, Corning, Corning, NY, USA). For the migration assay, $5 \times 10^{4}$ cells were added to the top chambers. For the invasion assay, $2 \times 10^{5}$ cells were seeded into the top chamber coated with Matrigel (BD Biosciences). Complete medium was added to the bottom wells to stimulate migration or invasion. After incubation for $24-48 \mathrm{~h}$, cells that did not penetrate through the membrane were removed with a cotton swab, while those adhered to the lower surface of the membrane were stained with $0.1 \%$ Crystal Violet. Five randomly selected fields per filter were counted.

Mammosphere-formation assays. Single-cell suspension was plated in the ultralow attachment six-well plates (Corning) at a density of $10000 \mathrm{cells} / \mathrm{ml}$ and grown in DMEM/F12 medium (serum free) supplemented with $20 \mu / / \mathrm{ml}$ B27 (Invitrogen), $20 \mathrm{ng} / \mathrm{ml} \mathrm{EGF} \mathrm{and} 20 \mathrm{ng} / \mathrm{ml} \mathrm{bFGF}$ at $37^{\circ} \mathrm{C}$ and $5 \% \mathrm{CO}_{2}$. Fresh media was added every 3 days. Mammospheres $>50 \mu \mathrm{m}$ in diameter were counted at day 8 .

Flow cytometry. A total of $1 \times 10^{6}$ cells were distributed in $100 \mu \mathrm{l}$ PBS containing $2 \%$ FBS in each test tube and kept on ice for $10 \mathrm{~min}$. The antibodies against CD44-APC and CD24-PE (BD Biosciences) were added to cell suspensions, incubated on ice and protected from light for $30 \mathrm{~min}$. Cells were washed and resuspended in $500 \mu$ FACS buffer and analyzed using a Flow Cytometer (BD Biosciences).

Luciferase reporter assay. HEK293T cells grown in 24-well plates were co-transfected with $50 \mathrm{ng}$ of psiCHECK2 (Promega) and miRNA mimics (GenePharma, Shanghai, China) using Lipofectamine2000 (Invitrogen). Fortyeight hours after transfection, luciferase activity was monitored using the DualLuciferase Reporter Assay System (Promega) and a luminometer (Molecular Devices, Sunnyvale, CA, USA). The Renilla activity was normalized to the firefly luciferase activity.

BrdU incorporation assay. BrdU incorporation assay was applied to measure the cell proliferation. BrdU pulses were performed in MCF10A and MDAMB-231 for $8 \mathrm{~h}(4 \mu \mathrm{g} / \mathrm{ml})$ and then tested by BrdU antibody (BD Biosciences) using flow cytometry.

RIP assay and RNA pull-down assay. The RIP experiment was carried out with the EZ-Magna RIP Kit (Millipore) according to the manufacturer's protocol using $5 \mu \mathrm{g}$ of goat anti-AGO2 antibody (Santa Cruz). The Flag-MS2bp- 
MS2bs-based RIP assay was carried out by using the ANTI-FLAG M2 Affinity Gel (Sigma). The co-precipitated RNAs were extracted with trizol reagent (Invitrogen) and detected by RT-PCR or qRT-PCR. RNA pull-down assay was carried out by using Flag-MS2bp-MS2bs-based pull-down assay. Specifically, pcDNA3-FlagMS2bp and pcDNA3-ROR-MS2bs were co-transfected to 293T cells, and the cells were harvested after $48 \mathrm{~h}$. About $1 \times 10^{7}$ cells were lysed in the soft lysis buffer (20 mM Tris- $\mathrm{Cl}, \mathrm{pH} 8.0,10 \mathrm{mM}$ Nacl, $1 \mathrm{mM}$ EDTA, 0.5\% NP-40) supplemented with RNasin (80 units/ml, Promega). Fifty microlitres of ANTI-FLAG M2 Magnetic Beads was added to each binding reaction tube and incubated at $4{ }^{\circ} \mathrm{C}$ for $4 \mathrm{~h}$. Beads were washed five times with the lysis buffer and boiled in $1 \times$ loading buffer for $10 \mathrm{~min}$. The retrieved proteins were subjected to SDS-PAGE and detected by western blotting.

In vivo tumor formation and lung-colonization assays. MDA-MB231 cells stably expressing the control shRNA or the linc-ROR-specific shRNA $\left(5 \times 10^{6}\right)$ were subcutaneously injected into the flanks of nude mice. The mice were killed at week 8 , and tumors were surgically isolated. Tumors were photographed and weighted later. The nude mice were injected with either the control or the linc-ROR-knockdown MDA-MB-231 cells $\left(1.5 \times 10^{6}\right)$ into the tail veins. Lungs were subsequently collected at week 9 and fixed in $10 \%$ formalin. The number of metastatic colonies on the surface of the lungs was counted under a dissection microscope, and then the lung tissue sections were stained with hematoxylin and eosin. All animal experiments were approved by the Animal Care Committee of the Northeast Normal University, China.

Statistical analysis. Data are presented as mean \pm S.D. The Paired $t$-test was used to determine the statistical significance of differences between groups. $P<0.05$ was considered statistically significant. The number of mice that developed lung metastases was analyzed by $\chi^{2}$-test. Statistical analysis was performed using the GraphPad Prism software (GraphPad Software, La Jolla, CA, USA).

\section{Conflict of Interest}

The authors declare no conflict of interest.

Acknowledgements. We thank Dr. Sabine Loewer (Children's Hospital Boston), and Dr. Xiaofei Zheng (Academy of Military Medical Sciences). This work was supported by the grants from the National Natural Science Foundation of China (grant numbers 31071149, 31170719, 31371294, 31100998, 31271442).

1. Ferlay J, Shin HR, Bray F, Forman D, Mathers C, Parkin DM. Estimates of worldwide burden of cancer in 2008: GLOBOCAN 2008. Int J Cancer 2010; 127: 2893-2917.

2. Zhao P, Dai M, Chen W, Li N. Cancer trends in China. Jpn J Clin Oncol 2010; 40: 281-285.

3. Thiery JP. Epithelial-mesenchymal transitions in development and pathologies. Curr Opin Cell Biol 2003; 15: 740-746.

4. Thiery JP, Acloque H, Huang RY, Nieto MA. Epithelial-mesenchymal transitions in development and disease. Cell 2009; 139: 871-890.

5. Baum B, Settleman J, Quinlan MP. Transitions between epithelial and mesenchymal states in development and disease. Semin Cell Dev Biol 2008; 19: 294-308.

6. Frith MC, Bailey TL, Kasukawa T, Mignone F, Kummerfeld SK, Madera M et al. Discrimination of non-protein-coding transcripts from protein-coding mRNA. RNA Biol 2006; 3: 40-48.

7. Wang KC, Chang HY. Molecular mechanisms of long noncoding RNAs. Mol Cell 2011; 43 : 904-914.

8. Ponting CP, Oliver PL, Reik W. Evolution and functions of long noncoding RNAs. Cell 2009; 136: 629-641.

9. Guttman M, Donaghey J, Carey BW, Garber M, Grenier JK, Munson G et al. lincRNAs act in the circuitry controlling pluripotency and differentiation. Nature 2011; 477 : 295-300.

10. Gupta RA, Shah N, Wang KC, Kim J, Horlings HM, Wong DJ et al. Long non-coding RNA HOTAIR reprograms chromatin state to promote cancer metastasis. Nature 2010; 464: 1071-1076.

11. Tsai MC, Manor O, Wan Y, Mosammaparast N, Wang JK, Lan F et al. Long noncoding RNA as modular scaffold of histone modification complexes. Science 2010; 329: 689-693.

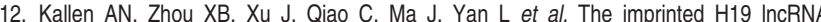
antagonizes let-7 microRNAs. Mol Cell 2013; 52: 101-112.

13. Kumar MS, Armenteros-Monterroso E, East P, Chakravorty P, Matthews N, Winslow MM et al. HMGA2 functions as a competing endogenous RNA to promote lung cancer progression. Nature 2013; 505: 212-217.
14. Guttman M, Amit I, Garber M, French C, Lin MF, Feldser D et al. Chromatin signature reveals over a thousand highly conserved large non-coding RNAs in mammals. Nature 2009; 458: 223-227.

15. Wapinski O, Chang HY. Long noncoding RNAs and human disease. Trends Cell Biol 2011; 21: 354-361.

16. Loewer S, Cabili MN, Guttman M, Loh YH, Thomas K, Park IH et al. Large intergenic non-coding RNA-RoR modulates reprogramming of human induced pluripotent stem cells. Nat Genet 2010; 42: 1113-1117.

17. Zhang A, Zhou N, Huang J, Liu Q, Fukuda $K$, Ma D et al. The human long non-coding RNA-RoR is a p53 repressor in response to DNA damage. Cell Res 2013; 23: 340-350.

18. Wang $Y, X u Z$, Jiang $J, X u C$, Kang $J$, Xiao $L$ et al. Endogenous miRNA sponge lincRNARoR regulates Oct4, Nanog, and Sox2 in human embryonic stem cell self-renewal. Dev Cell 2013; 25: 69-80.

19. Gregory PA, Bert AG, Paterson EL, Barry SC, Tsykin A, Farshid G et al. The miR-200 family and miR-205 regulate epithelial to mesenchymal transition by targeting ZEB1 and SIP1. Nat Cell Biol 2008; 10: 593-601.

20. Yu $F$, Yao H, Zhu $P$, Zhang $X$, Pan $Q$, Gong $C$ et al. let-7 regulates self renewal and tumorigenicity of breast cancer cells. Cell 2007; 131: 1109-1123.

21. Bareiss PM, Paczulla A, Wang H, Schairer R, Wiehr S, Kohlhofer U et al. SOX2 expression associates with stem cell state in human ovarian carcinoma. Cancer Res 2013; 73: $5544-5555$

22. Kumar SM, Liu S, Lu H, Zhang H, Zhang PJ, Gimotty PA et al. Acquired cancer stem cell phenotypes through Oct4-mediated dedifferentiation. Oncogene 2012; 31: 4898-4911.

23. Takahashi K, Yan IK, Haga H, Patel T. Modulation of hypoxia-signaling pathways by extracellular long non-coding RNA regulator of reprogramming. J Cell Sci 2014; 127(Pt 7): 1585-1594.

24. Mani SA, Guo W, Liao MJ, Eaton EN, Ayyanan A, Zhou AY et al. The epithelialmesenchymal transition generates cells with properties of stem cells. Cell 2008; 133: 704-715.

25. Wu H, Zhu S, Mo YY. Suppression of cell growth and invasion by miR-205 in breast cancer. Cell Res 2009; 19: 439-448.

26. Yang S, Li Y, Gao J, Zhang T, Li S, Luo A et al. MicroRNA-34 suppresses breast cancer invasion and metastasis by directly targeting Fra-1. Oncogene 2013; 32: 4294-4303.

27. Li L, Yuan L, Luo J, Gao J, Guo J, Xie X. MiR-34a inhibits proliferation and migration of breast cancer through down-regulation of Bcl-2 and SIRT1. Clin Exp Med 2013; 13: 109-117.

28. Nelson PT, Hatzigeorgiou AG, Mourelatos Z. miRNP:mRNA association in polyribosomes in a human neuronal cell line. RNA 2004; 10: 387-394.

29. Izaurralde $E$. Elucidating the temporal order of silencing. EMBO Rep 2012; 13: 662-663.

30. Cesana M, Cacchiarelli D, Legnini I, Santini T, Sthandier O, Chinappi M et al. A long noncoding RNA controls muscle differentiation by functioning as a competing endogenous RNA. Cell 2011; 147: 358-369.

31. Perez-Pomares JM, Munoz-Chapuli R. Epithelial-mesenchymal transitions: a mesodermal cell strategy for evolutive innovation in Metazoans. Anat Rec 2002; 268: 343-351.

32. Sleeman KE, Kendrick H, Ashworth A, Isacke CM, Smalley MJ. CD24 staining of mouse mammary gland cells defines luminal epithelial, myoepithelial/basal and non-epithelial cells. Breast Cancer Res 2006; 8: R7.

33. Yang J, Mani SA, Donaher JL, Ramaswamy S, Itzykson RA, Come C et al. Twist, a master regulator of morphogenesis, plays an essential role in tumor metastasis. Cell 2004; 117: 927-939

34. Peinado H, Olmeda D, Snail Cano A. Zeb and bHLH factors in tumour progression: an alliance against the epithelial phenotype? Nat Rev Cancer 2007; 7: 415-428.

35. Mani SA, Yang J, Brooks M, Schwaninger G, Zhou A, Miura N et al. Mesenchyme Forkhead 1 (FOXC2) plays a key role in metastasis and is associated with aggressive basal-like breast cancers. Proc Natl Acad Sci USA 2007; 104: 10069-10074.

36. Pauli A, Valen E, Lin MF, Garber M, Vastenhouw NL, Levin JZ et al. Systematic identification of long noncoding RNAs expressed during zebrafish embryogenesis. Genome Res 2012; 22: 577-591.

37. Ng SY, Johnson R, Stanton LW. Human long non-coding RNAs promote pluripotency and neuronal differentiation by association with chromatin modifiers and transcription factors. EMBO J 2012; 31: 522-533.

38. Gabory A, Jammes H, Dandolo L. The H19 locus: role of an imprinted non-coding RNA in growth and development. Bioessays 2010; 32: 473-480.

39. Onyango $\mathrm{P}$, Feinberg AP. A nucleolar protein, $\mathrm{H} 19$ opposite tumor suppressor (HOTS), is a tumor growth inhibitor encoded by a human imprinted $\mathrm{H} 19$ antisense transcript. Proc Natl Acad Sci USA 2011; 108: 16759-16764

40. Lottin S, Adriaenssens E, Dupressoir T, Berteaux N, Montpellier C, Coll J et al. Overexpression of an ectopic $\mathrm{H} 19$ gene enhances the tumorigenic properties of breast cancer cells. Carcinogenesis 2002; 23: 1885-1895.

41. Matouk IJ, DeGroot N, Mezan S, Ayesh S, Abu-lail R, Hochberg A et al. The H19 non-coding RNA is essential for human tumor growth. PLoS One 2007; 2: e845.

42. Luo M, Li Z, Wang W, Zeng Y, Liu Z, Qiu J. Upregulated H19 contributes to bladder cancer cell proliferation by regulating ID2 expression. FEBS J 2013; 280: 1709-1716.

43. Kaneko S, Li G, Son J, Xu CF, Margueron R, Neubert TA et al. Phosphorylation of the PRC2 component Ezh2 is cell cycle-regulated and up-regulates its binding to ncRNA. Genes Dev 2010; 24: 2615-2620. 
44. Nagano T, Mitchell JA, Sanz LA, Pauler FM, Ferguson-Smith AC, Feil R et al. The Air noncoding RNA epigenetically silences transcription by targeting G9a to chromatin. Science 2008; 322: 1717-1720.

45. Izaguirre DI, Zhu W, Hai T, Cheung HC, Krahe R, Cote GJ. PTBP1-dependent regulation of USP5 alternative RNA splicing plays a role in glioblastoma tumorigenesis. Mol Carcinog 2012; 51: 895-906.

46. Ohno S, Shibayama M, Sato M, Tokunaga A, Yoshida N. Polypyrimidine tract-binding protein regulates the cell cycle through IRES-dependent translation of CDK11(p58) in mouse embryonic stem cells. Cell Cycle 2011; 10: 3706-3713.

47. He X, Pool M, Darcy KM, Lim SB, Auersperg N, Coon JS et al. Knockdown of polypyrimidine tract-binding protein suppresses ovarian tumor cell growth and invasiveness in vitro. Oncogene 2007; 26: 4961-4968.

48. He X, Arslan AD, Ho TT, Yuan C, Stampfer MR, Beck WT. Involvement of polypyrimidine tract-binding protein (PTBP1) in maintaining breast cancer cell growth and malignant properties. Oncogenesis 2014; 3 : e84.

49. Al-Hajj M, Wicha MS, Benito-Hernandez A, Morrison SJ, Clarke MF. Prospective identification of tumorigenic breast cancer cells. Proc Natl Acad Sci USA 2003; 100: 3983-3988.

50. Peinado $\mathrm{H}$, Portillo F, Cano A. Transcriptional regulation of cadherins during development and carcinogenesis. Int J Dev Biol 2004; 48: 365-375.
51. Park SM, Gaur AB, Lengyel E, Peter ME. The miR-200 family determines the epithelial phenotype of cancer cells by targeting the E-cadherin repressors ZEB1 and ZEB2. Genes Dev 2008; 22: 894-907.

52. Tellez CS, Juri DE, Do K, Bernauer AM, Thomas CL, Damiani LA et al. EMT and stem cell-like properties associated with miR-205 and miR-200 epigenetic silencing are early manifestations during carcinogen-induced transformation of human lung epithelial cells. Cancer Res 2011; 71: 3087-3097.

(c) (i) (2) Cell Death and Disease is an open-access journal published by Nature Publishing Group. This work is licensed under a Creative Commons Attribution-NonCommercialShareAlike 3.0 Unported License. The images or other third party material in this article are included in the article's Creative Commons license, unless indicated otherwise in the credit line; if the material is not included under the Creative Commons license, users will need to obtain permission from the license holder to reproduce the material. To view a copy of this license, visit http://creativecommons.org/ licenses/by-nc-sa/3.0/

Supplementary Information accompanies this paper on Cell Death and Disease website (http://www.nature.com/cddis) 